\title{
Paradigm Shift of HRM Practices in the Banking Sector of Bangladesh
}

\author{
Nusrat Fatema \\ (Lecturer, Department of Business Administration, Stamford University Bangladesh, Bangladesh)
}

\begin{abstract}
This paper mainly focused on how the paradigm of HRM practices in the banking industry of Bangladesh shifted from decades to decades. For the research purpose, we have divided the banking sector into - Public and Private Banks; conducted a qualitative research and compared the actual result of the HRM practices in both types of banks. The first section of this report covered the actual situation of HRM practices in Bangladesh, based on research opinion of various authors concerning the vital issues of HRM like - Recruiting, Selection, Training \& Development, Compensation \& Benefit, Performance Appraisal, Promotion and Industrial Relation. The research methodology was comprised of two methods - Primary Method (Questionnaire survey and in-depth Interview) and Secondary Method (Online journals, Websites). Moreover, the questionnaire was divided into 3 decades to find exactly how the paradigm of HR shifted in the banking industry. Next section reveals the result \& discussion part based on the information given by the banks. From the findings the actual paradigm shift of HRM practices in the banking industry was portrayed in the next section which was followed by proper justification. Then the paper was concluded with some unique suggestions to improvise the HRM practice in the banking sector.
\end{abstract}

Keywords: Compensation, Human Resource Management, Performance Appraisal, Recruitment, Selection.

\section{Introduction}

Bangladeshi workforce is labor intensive. Large portion of workmen are semi skilled and unskilled. Today, Bangladesh is pursuing a liberal market economy and it has encouraged increased participation of the private sector and export oriented industries. Moreover, large investments into the economy are coming in the form of joint ventures and foreign direct investments (FDI) by the establishment of multinational companies (MNC). Despite of the investments from different organizations and MNCs, there is acute shortage of Knowledge \& Skill-based workers in Bangladesh [1].

In any particular nation, HRM (Human Resource Management) practices are rooted in the historical, political, social and political differences of the country [2]. HRM in Bangladesh has been shaped by several factors ranging from historical, political, economical, as well as cultural and institutional factors. In a survey it was found that due to the outdated HR practices, the productivity and motivation level of the employees of Bangladesh are very low [3] .

According to Hofstede's cultural dimension, Bangladesh predominantly belongs to the hierarchical society and the culture is characterized by a high degree of power distance, collectivism and low performance orientation which directly influence the practice of HRM within the organizations [4]. Subordinates feel comfortable to work under superiors and superior-subordinate relationships are characterized by protection, patronage and favor on the part of the superiors and respect, loyalty and compliance on the part of the subordinates. Deviant behavior and ideas are less encouraged. Opposing a superior's decisions or raising a question is often considered ill mannered [5].

Bangladesh, by virtue of its social set-up, encourages extended families built on strong relationship. An "extended family" culture in Bangladesh influences recruitment. In such a set up, there is a tendency to prefer recruits who come through known contacts within the system. Newspapers continue to be the most commonly used source of recruitment. However, internal job posting is prevalent in Bangladesh. A large number of organizations fill vacancies at middle and senior levels from existing employee pool, significant importance is not attached to the process of succession planning. Governmental organizations in Bangladesh have become almost dysfunctional as being filled by unethical act. In most training sessions and workshops people not deserving them are given the opportunity. This may be because of family ties, political influence or just merely being the favorite of the boss [6].

In Bangladesh, many organizations have been following the same compensation practices since long. Since the per capita income is not very high, organisations do not risk experimenting with the earning of individuals. In a book review it was mentioned that compensation is a very important aspect of HRM in Bangladesh where employees are commonly low paid with little or no fringe benefits [7]. A study found that most of the surveyed organizations assign compensation management and payroll handling responsibilities to specific sub-departments (accounts / finance) [8]. Outsourcing is a rare phenomenon in Bangladesh since 
organisations have in house payroll processing. Inflation, cost of living, individual performance are the key determinants for annual increments. The determination of compensation is based on the industry standards, not based on economic measurements (inflation, CPI) and living standards. Sometimes, organisation determines the compensation based on individual performance, but without having an actual measurement of the performance.

Performance appraisal has attracted a great deal of attention. Annual performance appraisals enable management to monitor the standards, to agree on the expectations and objectives, and to delegate the responsibilities and tasks [9]. A study of HRM practices of 92 medium and large business enterprises in Bangladesh found that only $62 \%$ of surveyed organizations had an HR/IR (industrial relation) department. About $96 \%$ organizations had training programs. $91 \%$ of organizations had performance appraisal system and similar percent of organizations had a system of rewarding the good employees [10].

It was also found that trade unions of Bangladesh are unorganized, powerless, lack spontaneous workers support, highly politicized, and the members are not efficient enough to raise voice for the workers [11]. Many entrepreneurs, and employers do not believe in industrial relation, rather they like treating workers as the most neglected component of production, use the bad technique of managing the leaders rather than encouraging trade union for industrial relation. In short, industrial relation situation in Bangladesh is not encouraging. The Bangladesh Labor Act 2006, which is a major and comprehensive enactment, has not been able to bring the desired changed as a response to demand of stakeholders for improving the regulatory framework on trade union due to its in-built weaknesses, and lack of institutional capacity to implement the laws [12].

The banking sector of Bangladesh relative to the size of its economy is comparatively larger than many economies of similar level of development and per capita income. The total size of the sector is $26.54 \%$ of GDP, which is proportionately large for a country with a per capita income of only about US\$370 [13].

Banking Industry is a potential industry on which the growth of a country depends a lot. In Bangladesh banking industry is also very influential. In Bangladesh, this industry holds a lot of skilled employees. Human Resource Management (HRM) is very much applicable and well practiced in the banks of Bangladesh. Through the study of the paradigm shift in the HRM practices in the banking sector of Bangladesh, the research aims not to only fill the dearth of the literatures found in these areas but also to create inroads for understanding the perspective of Bangladeshi management.

\section{II. banking industry at a glance}

TABLE 1: Background of banking Industry - Sonali , Janata, National Bank Ltd., Mutual Trust Bank Ltd.

\begin{tabular}{|c|c|c|c|c|}
\hline Industry types & \multicolumn{4}{|l|}{ Banking Industry } \\
\hline Banking & Company A & Company B & Company $\mathrm{C}$ & Company D \\
\hline Company & Sonali Bank & Janata Bank & National Bank & Mutual Trust Bank Ltd \\
\hline Interviews & $\begin{array}{lr}\begin{array}{l}\text { Mrs. } \\
\text { Khanom, } \\
\text { officer }\end{array} & \text { Khairunnesa } \\
\end{array}$ & $\begin{array}{l}\text { Mr. Kazi Jamaluddin, Ex - } \\
\text { principal officer }\end{array}$ & $\begin{array}{lr}\text { Mr.Abdul } & \text { wadud } \\
\text { Management } & \text { trainee } \\
\text { officer } & \end{array}$ & $\begin{array}{l}\text { Mr. A. B. M. Shamsur } \\
\text { Rahman, Executive Vice } \\
\text { President }\end{array}$ \\
\hline Main product & $\begin{array}{l}\text { all traditional banking } \\
\text { functions including } \\
\text { deposit mobilization } \\
\text { and lending \& treasury } \\
\text { functions as the agent } \\
\text { of the BB }\end{array}$ & $\begin{array}{l}\text { Retail/Personal Banking, } \\
\text { Credit programs, Micro } \\
\text { Enterprises \& Special Credit, } \\
\text { Rural Banking / Credit } \\
\text { Program, International } \\
\text { Banking, Foreign Remittance } \\
\text { and NRB Banking }\end{array}$ & $\begin{array}{lr}\text { All types of } \\
\text { Commercial } & \text { Banking } \\
\text { Activities } & \text { including } \\
\text { Money } & \text { Market } \\
\text { operations } & \text { to Any } \\
\text { related } & \text { Financial } \\
\text { Services. } & \end{array}$ & $\begin{array}{l}\text { All types of Commercial } \\
\text { Banking Activities } \\
\text { including Money Market } \\
\text { operations to Any } \\
\text { related } \\
\text { Services. Financial }\end{array}$ \\
\hline $\begin{array}{l}\text { Establishment } \\
\text { year }\end{array}$ & 1972 & 1972 & 1983 & 1999 \\
\hline $\begin{array}{l}\text { Number of } \\
\text { Employees }\end{array}$ & 26,085 & $15109\left(1^{\text {st }}\right.$ class to $4^{\text {th }}$ class $)$ & $\begin{array}{l}2239, \quad(1689 \\
\text { officers/executives, } \\
550 \text { staff. })\end{array}$ & $\begin{array}{l}2845 \text { (permanent - } \\
1245 \text {, temporary }-1600)\end{array}$ \\
\hline $\begin{array}{l}\% \text { foreign } \\
\text { Capital }\end{array}$ & $0 \%$ & $0 \%$ & $25 \%$ & $0 \%$ \\
\hline $\begin{array}{l}\text { No of foreign } \\
\text { expertise }\end{array}$ & $\begin{array}{l}\text { Not applicable as } \\
\text { national banks }\end{array}$ & $\begin{array}{l}\text { Not applicable as sate owned } \\
\text { bank }\end{array}$ & $\begin{array}{l}\text { Not hired any foreign } \\
\text { expertise }\end{array}$ & Not hired \\
\hline $\begin{array}{l}\text { Total Asset } \\
\text { (US\$) }\end{array}$ & US\$ 299.15 million & US\$4890.756 million & US\$ 1.7 billion & US\$ 753.93 million \\
\hline $\begin{array}{l}\% \quad \text { Sale in } \\
\text { domestic } \\
\text { Market }\end{array}$ & $21.2 \%$ & $34 \%$ & $41.26 \%$ & $33 \%$ \\
\hline $\begin{array}{l}\% \text { Of Overseas } \\
\text { Market }\end{array}$ & $25.87 \%$ & $25 \%$ & $24.42 \%$ & $18 \%$ \\
\hline Average Age & $\begin{array}{l}45+\text { (assumption), } 28 \\
\text { years }\end{array}$ & $45+$ (assumption), 25 years & $\begin{array}{l}45+(\text { assumption }), 25 \\
\text { years }\end{array}$ & $\begin{array}{l}40+\text { (assumption), } 25 \\
\text { years }\end{array}$ \\
\hline $\begin{array}{l}\text { Average } \\
\text { Education }\end{array}$ & MBA, Masters & MBA, Masters & MBA, Masters & MBA, Masters \\
\hline
\end{tabular}


Paradigm Shift of HRM Practices in the Banking Sector of Bangladesh

\begin{tabular}{|c|c|c|c|c|}
\hline $\begin{array}{l}\text { Average } \\
\text { monthly salary }\end{array}$ & 55000 & 50000 & 90000 & $\begin{array}{l}100000, \\
\text { managerial } \\
70000)\end{array}$ \\
\hline $\begin{array}{l}\text { Number of } \\
\text { Strike Day }\end{array}$ & Approximately & $\begin{array}{l}\text { Not seen in managerial } \\
\text { position }\end{array}$ & Not seen any strike & $\begin{array}{l}\text { Not seen in managerial } \\
\text { position }\end{array}$ \\
\hline $\begin{array}{l}\text { Business } \\
\text { Growth }\end{array}$ & $17.3 \% \%$ & $14 \%$ & $20 \%$ & $15 \%$ \\
\hline Sales Growth & $51 \%$ & $40 \%$ & $33.37 \%$ & $44 \%$ \\
\hline $\begin{array}{l}\text { Employee } \\
\text { Turnover Rate }\end{array}$ & $1 \%$ & $1 \%$ & $2 \%$ & $2 \%$ \\
\hline
\end{tabular}

Sources: [14] [15] [16] [17] [18] [19] [20].

Table 1 shows the overall progress of all the four banks in terms of performance, efficiency, and employment, business and service expansion for nearly about three decades. Overall sales growth rate has increased as the benefit and service options are increasing for customers, for example- Online banking option is introduced in private banks, but public banks could not introduce this facility yet in most of their banking zones except for Dhaka city. However the Business growth, Market share and assets are comparatively decreasing per period in both the public and private banks and it is rule of the industry. On the contrary, public banks are more powerful in certain criterion, for example - Sonali Bank singularly enjoys the prestige of being the agent of the Central Bank of Bangladesh in such places where the guardian of the money market does not act by itself. The bank discharges the treasury functions as the agent of the Bangladesh Bank and it collects tax, stamp duty and registration fees, operates special savings accounts, pays salaries to the teachers of schools, and colleges and pension to retired government employees. Moreover it provides funding to some income generating and economic development projects, such as Poverty Alleviation Credit Program, Female Special Credit Program, and Agro-based Industrial Credit Program in the rural areas and backed by the Government. People feel safe while dealing with public banks but it is now easier to get service from the private banks across the country and world. Thus more and more competition is headed in both sectors and increases the quality services. But in terms of salary payment structure of public banks are much lower than the private banks which, negatively affects the motivation level of employees. Despite the dissatisfactory compensation level, due to additional benefit differences, employee turnover rate is very low as public banks job security is higher and they provide more employee benefits and compensation then the private banks, though job enrichment facility is still not updated in public banks. Public Banks are now capable of preventing the strike which was first observed in the 1980 or 1984 in the public banks where as private banks are free from this problem. Unfortunately public banks do not want to reveal their number of strike days since managerial positions did not take any initiative in strikes as well as not involved in the labor union.

\section{Research Methodology:}

The prime objective of this study was to discover and analyze the paradigm shift of HRM practices in the banking industry of Bangladesh. For this purpose four well known and respected banks were selected among which two are from public side and two are from private sector. Respective Public banks are Sonali Bank, Janata Bank; and the Private Banks are - National Bank, Mutual Trust bank Ltd. All the banks were selected based on their reputation, market position, revenue, sales growth and so on. For the purpose of excavating the actual information two types of sources were used in our project and those are primary sources and secondary sources.

\subsection{Primary Sources}

To collect the primary data, Questionnaire survey and in-depth interviews were conducted. The objective of this survey was to pretest the items and to fine tune the items and construct measurement. The research instrument (questionnaire) was designed to find out qualitative data on the paradigm shift of HRM in Private \& Public banks of Bangladesh.

\subsubsection{Questionnaire survey}

The questionnaire engaged 7 respondent executives and managers based on age and position as well as the length of the service from these banking sectors and picked randomly. This also included managers those we interviewed to find more information. The sample respondents were selected at random basis from our selected Banks. The HRM practices with 8 variables like - Recruitment, Selection, Training Development, Promotion, Performance Appraisal, Compensation, Benefits \& Services, and Industrial Relations Criteria were tested with these managers to obtain feedback, and assess the scale validity of the survey. The decade's were also divided according to 1971-1980, 1980-1990, and 1991-2010, so that we could identify the actual paradigm shift, as the growth of HRM practices could be shown best in this way. The questionnaires were distributed and collected in person, which allowed the surveyors to ask relevant questions to the sample respondents to gain some additional 
insight and feedback on the survey instrument; as a result the construct validity of the questionnaire survey was obtained. The response rate was satisfactory. Each respondent spent about 25 minutes to 30 minutes to complete the questionnaire.

\subsubsection{In-depth Interviews}

The second stage which was the main source of the information was the in-depth interviews. The objectives of in-depth interviews were to investigate personal, sensitive and confidential information related to human resource management practices and their implications in Private \& Public banks for improving the performance. For that reason semi structured interviews were conducted with four respectable employees of these four banks. This semi structured interviews start with open, general questions and then focus more on the specifics of the identified research issues [21] [22]. The appointments for the in-depth interviews were made via telephone conversation. Each interview began with a general introduction to acquaint the respondent with the interview purpose and agenda [23] [24]. Throughout the interview, respondents were allowed to tell their experiences in their own words without any prompting or input from the researcher. This research section deals with specific research questions. In addition, the result of the interviews was shown to the respondents, for checking on errors and adding information as necessary.

\subsection{Secondary Sources}

For secondary data collection a range of websites containing latest information about the financial institutions of Bangladesh and also information related to overall human resource practices in the context of Bangladesh is been used for this research report. Some online journals written by several research persons, their experience, surveys and personal opinions also remain helpful and useful in developing this report.

\section{Results of paradigm shift of Human Resource Management Practices}

In this section, all the data obtained from the primary sources for each of the variable of HRM practice are set down in a table format. In addition to that, any distinctive feature which was found from those is also described precisely.

\begin{tabular}{|c|c|c|c|c|c|c|c|}
\hline \multirow{2}{*}{\multicolumn{2}{|c|}{$\begin{array}{l}\text { Recruitment } \\
\text { criteria }\end{array}$}} & \multirow{2}{*}{\multicolumn{2}{|c|}{$\begin{array}{l}\text { 1971-1980 } \\
\text { Overall Industries }\end{array}$}} & \multirow{2}{*}{\multicolumn{2}{|c|}{$\begin{array}{l}\text { 1981-1990 } \\
\text { Overall Industries }\end{array}$}} & \multirow{2}{*}{\multicolumn{2}{|c|}{$\begin{array}{l}\text { 1991-2010 } \\
\text { Overall Industries }\end{array}$}} \\
\hline & & & & & & & \\
\hline & & \multirow{2}{*}{$\begin{array}{l}\text { Public } \\
\text { Newspaper. }\end{array}$} & \multirow{2}{*}{$\begin{array}{l}\text { P } \\
\text { v } \\
\text { t. }\end{array}$} & \multirow{2}{*}{$\begin{array}{l}\text { Public } \\
\text { Newspaper }\end{array}$} & \multirow{2}{*}{$\begin{array}{l}\text { Private } \\
\text { Newspaper }\end{array}$} & \multirow{2}{*}{ Public } & \multirow{2}{*}{$\begin{array}{l}\text { Private } \\
\text { Newspaper }\end{array}$} \\
\hline 1 & $\begin{array}{l}\text { Advertiseme } \\
\text { nt in the } \\
\text { media }\end{array}$ & & & & & & \\
\hline 2 & $\begin{array}{l}\text { Advertiseme } \\
\mathrm{nt} \text { in the } \\
\text { notice board }\end{array}$ & Did not practice this & & $\begin{array}{l}\text { No advertisement } \\
\text { in notice board }\end{array}$ & Not practiced & $\begin{array}{l}\text { Did not use } \\
\text { this }\end{array}$ & Not used \\
\hline 3 & $\begin{array}{l}\text { Employment } \\
\text { agencies }\end{array}$ & $\begin{array}{l}\text { Not used as } \\
\text { recruitment is done } \\
\text { by the bank itself or } \\
\text { Bangladesh Bank }\end{array}$ & & $\begin{array}{ll}\text { Not used as } & \text { BR } \\
\text { Committee } & \text { do } \\
\text { recruiting } & \end{array}$ & Not used & $\begin{array}{l}\text { Did not use } \\
\text { external } \\
\text { agency }\end{array}$ & Not used this \\
\hline 4 & Head hunting & $\begin{array}{l}\text { Personal } \\
\text { interviewwith } \\
\text { director. }\end{array}$ & & No head hunting & Did not applied & $\begin{array}{l}\text { Did not do } \\
\text { head hunting }\end{array}$ & $\begin{array}{l}\text { Never used } \\
\text { it }\end{array}$ \\
\hline 5 & $\begin{array}{l}\text { Professional } \\
\text { associations }\end{array}$ & Not recruit through it & & Not used & $\begin{array}{l}\text { Not hired } \\
\text { through it }\end{array}$ & $\begin{array}{l}\text { Did not } \\
\text { practice }\end{array}$ & Not used \\
\hline 6 & $\begin{array}{l}\text { Recommenda } \\
\text { tions }\end{array}$ & $\begin{array}{l}\text { Internal recruiting } \\
\text { was there (e.g. -non } \\
\text { managerial post) but } \\
\text { N/A for MTO, cash } \\
\text { officer. }\end{array}$ & & $\begin{array}{l}\text { Not practice for } \\
\text { MTO, cash officer } \\
\text { except the clerical } \\
\text { post }\end{array}$ & $\begin{array}{l}\text { Not performed } \\
\text { for cash officer, } \\
\text { but clerical post } \\
\text { its applicable }\end{array}$ & $\begin{array}{l}\text { Only } \\
\text { applicable for } \\
\text { non } \\
\text { managerial } \\
\text { post }\end{array}$ & $\begin{array}{l}\text { Yes for non } \\
\text { managerial } \\
\text { post, but not } \\
\text { for MTO } \\
\text { post. }\end{array}$ \\
\hline 7 & $\begin{array}{l}\text { Employee } \\
\text { referrals }\end{array}$ & $\begin{array}{l}\text { Applicable } \quad \text { for } \\
\text { clerical post. }\end{array}$ & & $\begin{array}{l}4^{\text {th }} \text { class people } \\
\text { recruited through } \\
\text { it }\end{array}$ & $\begin{array}{l}\text { N/A for MTO } \\
\text { post, but for non } \\
\text { managerial post }\end{array}$ & $\begin{array}{l}\text { Considerable } \\
\text { for clerical } \\
\text { post. }\end{array}$ & $\begin{array}{l}\text { For clerical } \\
\text { post }\end{array}$ \\
\hline 8 & $\begin{array}{l}\text { Curriculum } \\
\text { vitae }\end{array}$ & $\begin{array}{l}\text { Not applicable for } \\
\text { managerial and non } \\
\text { - managerial post }\end{array}$ & & $\begin{array}{l}\text { N/A for } \\
\text { Managerial post, } \\
\text { but applicable for } \\
\text { non - managerial } \\
\text { post }\end{array}$ & $\begin{array}{l}\text { Did not } \\
\text { considered the } \\
\text { stored CV much }\end{array}$ & $\begin{array}{l}\text { N/A for } \\
\text { Managerial } \\
\text { post, } \\
\text { applicable for } \\
\text { non } \\
\text { managerial } \\
\text { post }\end{array}$ & $\begin{array}{l}\text { Most of the } \\
\text { time they } \\
\text { call } \\
\text { applicant for } \\
\text { exam from } \\
\text { the posted } \\
\text { CV }\end{array}$ \\
\hline 9 & Internet & Not introduced & & Not introduced & Not introduced & $\begin{array}{l}\text { BDjobs, } \\
\text { company } \\
\text { website. }\end{array}$ & $\begin{array}{l}\text { BDjobs, } \\
\text { company } \\
\text { website }\end{array}$ \\
\hline
\end{tabular}


Recruitment Criteria: Public banks used Newspaper during 1971 to 1980; whereas private banks were not even existed in the industry revealed in table 2. From $1991-2010$, public and private banks started to use internet along with Newspaper. Initially Public banks lacked in this area due to the unavailability and unfamiliarity of internet usage. But now there has been a great improvement since, both public and private Banks allow applicant to apply online via their official website and circular posted in "BDJobs". If suitable post is available in the private company (e.g. MTO position), hr department notifies suitable candidates out of the online applications for test exam via e - mail, send letter or through phone call in this time period but this is not practiced in public banks. Another innovative idea is practiced by the public banks, which is head hunting where applicants went for a personal interview with the director of the bank and if they survived the viva, they got the job according to their qualification and this practice was done based on biasness and favoritism during 1971 1980. But this practice was no longer effective after 1980's. Nowadays Public banks do not prefer recommendations, employee referrals, curriculum vitae in recruiting people in managerial position, but consider this for non managerial position (e.g. junior officer), whereas private banks prefer this area most except for MTO position.

\begin{tabular}{|c|c|c|c|c|c|c|c|}
\hline \multirow{3}{*}{\multicolumn{2}{|c|}{ Selection criteria }} & \multirow{2}{*}{\multicolumn{2}{|c|}{$\begin{array}{c}1971-1980 \\
\text { Overall Industries }\end{array}$}} & \multirow{2}{*}{\multicolumn{2}{|c|}{$\begin{array}{c}1981-1990 \\
\text { Overall Industries }\end{array}$}} & \multirow{2}{*}{\multicolumn{2}{|c|}{$\begin{array}{c}\text { 1991-2010 } \\
\text { Overall Industries }\end{array}$}} \\
\hline & & & & & & & \\
\hline & & Public & Pvt. & Public & Private & Public & Private \\
\hline 1 & Initial screening & $\begin{array}{l}\text { Screening done } \\
\text { in the personal } \\
\text { interview }\end{array}$ & - & $\begin{array}{l}\text { In the viva and } \\
\text { from the } \\
\text { application }\end{array}$ & $\begin{array}{l}\mathrm{CV} \text { and } \\
\text { viva }\end{array}$ & $\begin{array}{l}\text { Through the } \\
\text { application }\end{array}$ & $\begin{array}{l}\text { Yes, in the } \\
\text { application } \\
\text { form \& CV }\end{array}$ \\
\hline 2 & $\begin{array}{l}\text { Employment } \\
\text { tests }\end{array}$ & $\begin{array}{l}\text { Yes written test } \\
\text { for MTO }\end{array}$ & - & $\begin{array}{l}\text { Yes written test } \\
\text { for MTO }\end{array}$ & $\begin{array}{l}\text { Yes for } \\
\text { MTO }\end{array}$ & $\begin{array}{l}\text { Yes written } \\
\text { test for MTO }\end{array}$ & $\begin{array}{l}\text { Yes written } \\
\text { test for MTO }\end{array}$ \\
\hline 3 & Interview & $\begin{array}{l}\text { Viva was the } \\
\text { major source of } \\
\text { recruiting }\end{array}$ & - & $\begin{array}{c}\text { Yes as a form of } \\
\text { Viva }\end{array}$ & Yes & $\begin{array}{l}\text { Selected } \\
\text { candidate face } \\
\text { interview. non } \\
\text { managerial } \\
\text { candidate } \\
\text { directly sit for } \\
\text { interview }\end{array}$ & $\begin{array}{c}\text { Selected } \\
\text { candidate face } \\
\text { interview. } \\
\text { Clerical } \\
\text { candidate } \\
\text { directly sit for } \\
\text { interview }\end{array}$ \\
\hline 4 & $\begin{array}{c}\text { Background } \\
\text { investigation \& } \\
\text { reference cheek }\end{array}$ & $\begin{array}{l}\text { They follow this } \\
\text { that time. }\end{array}$ & - & $\begin{array}{l}\text { They have rules } \\
\text { but did not } \\
\text { follow regularly }\end{array}$ & $\begin{array}{l}\text { Follow } \\
\text { leniently }\end{array}$ & $\begin{array}{l}\text { Did not follow } \\
\text { strictly }\end{array}$ & $\begin{array}{l}\text { Did not } \\
\text { follow }\end{array}$ \\
\hline 5 & $\begin{array}{c}\text { Physical / } \\
\text { medical } \\
\text { examination }\end{array}$ & Yes & - & Yes & Yes & Yes & Yes \\
\hline 6 & Hiring decision & $\begin{array}{l}\text { Based on the } \\
\text { results of } \\
\text { previous steps, } \\
\text { final hiring } \\
\text { decision is taken }\end{array}$ & - & $\begin{array}{l}\text { Final decision is } \\
\text { taken if the } \\
\text { medical test } \\
\text { result fulfill } \\
\text { company's } \\
\text { requirement. }\end{array}$ & $\begin{array}{l}\text { Influenced } \\
\text { by } \\
\text { previous } \\
\text { result \& } \\
\text { reference } \\
\text { except } \\
\text { MTO }\end{array}$ & $\begin{array}{l}\text { previous result } \\
\text { \& sometime } \\
\text { reference do } \\
\text { influence } \\
\text { decision }\end{array}$ & $\begin{array}{c}\text { Influenced by } \\
\text { previous } \\
\text { results \& } \\
\text { reference } \\
\text { greatly affect } \\
\text { except MTO }\end{array}$ \\
\hline 7 & $\begin{array}{l}\text { Completion of } \\
\text { application form }\end{array}$ & $\begin{array}{l}\text { Yes, need to } \\
\text { complete for } \\
\text { future reference }\end{array}$ & - & $\begin{array}{l}\text { complete for } \\
\text { future reference }\end{array}$ & Yes & Yes & $\begin{array}{l}\text { commonly } \\
\text { says the staff } \\
\text { form }\end{array}$ \\
\hline
\end{tabular}

Selection Criteria: Public banks followed direct recruitment through personal interview along with preliminary test for their Management trainee post, during 1971- 2010, described in Table 3. Between 1971- 1990 written exam questionnaire used to be formed by the government organization "Banker Recruitment Committee" only for 4 state owned banks, whereas private banks followed their own recruiting system. Private Banks recruited with the help of performance Management initially. Now each state owned bank as well as private banks recruit through HR department. Interview is important in every case, either for managerial position or for non managerial position in both banks, and reference influences the result mostly, especially in private banks. The interview session is conducted by reputed university faculty, managing director, and other respected person invited by the company. Both the banking industry follows same techniques in hiring for MTO. Now a day's public and private banks do not follow the background information checking for their selected candidates on a regular basis. 


\begin{tabular}{|c|c|c|c|c|c|c|c|}
\hline \multirow{3}{*}{\multicolumn{2}{|c|}{ Training criteria }} & \multirow{2}{*}{\multicolumn{2}{|c|}{$\begin{array}{c}1971-1980 \\
\text { Overall Industries }\end{array}$}} & \multirow{2}{*}{\multicolumn{2}{|c|}{$\begin{array}{c}\text { 1981-1990 } \\
\text { Overall Industries }\end{array}$}} & \multirow{2}{*}{\multicolumn{2}{|c|}{$\begin{array}{c}\text { 1991-2010 } \\
\text { Overall Industries }\end{array}$}} \\
\hline & & & & & & & \\
\hline & & \multirow{2}{*}{$\begin{array}{c}\text { Public } \\
\text { Mostly used } \\
\text { techniques }\end{array}$} & \multirow{2}{*}{$\begin{array}{c}\text { Private } \\
-\end{array}$} & \multirow{2}{*}{$\begin{array}{c}\text { Public } \\
\text { Used most }\end{array}$} & \multirow{2}{*}{$\begin{array}{c}\text { Private } \\
\text { Best used } \\
\text { techniques. }\end{array}$} & \multirow{2}{*}{$\begin{array}{c}\text { Public } \\
\text { Generally } \\
\text { used } \\
\text { techniques }\end{array}$} & \multirow{2}{*}{$\begin{array}{l}\text { Private } \\
\text { Best option for } \\
\text { learning. }\end{array}$} \\
\hline & $\begin{array}{l}\text { On-the-job } \\
\text { training }\end{array}$ & & & & & & \\
\hline 2 & $\begin{array}{l}\text { Classroom } \\
\text { lecture }\end{array}$ & $\begin{array}{l}\text { Formal } \\
\text { technique } \\
\text { (28 days ) }\end{array}$ & - & $\begin{array}{c}\text { Employees } \\
\text { went to } \\
\text { different } \\
\text { branches for } \\
\text { learning along } \\
\text { with CL(3 } \\
\text { months - } 28 \\
\text { days) }\end{array}$ & $\begin{array}{l}\text { mandatory } \\
\text { (training } \\
\text { length } 6 \\
\text { month ) }\end{array}$ & $\begin{array}{l}\text { highly used } \\
\text { technique }\end{array}$ & $\begin{array}{l}\text { highly used } \\
\text { technique (now } \\
\text { training length } \\
\text { reduced to } 45 \\
\text { days) }\end{array}$ \\
\hline 3 & Conference & $\begin{array}{c}\text { Not } \\
\text { introduced }\end{array}$ & - & $\begin{array}{c}\text { Not } \\
\text { introduced }\end{array}$ & $\begin{array}{c}\text { Not } \\
\text { introduced }\end{array}$ & $\begin{array}{c}\text { Not } \\
\text { introduced till } \\
\text { now }\end{array}$ & $\begin{array}{c}\text { Not introduced } \\
\text { yet. }\end{array}$ \\
\hline 4 & $\begin{array}{l}\text { Audiovisual } \\
\text { technique }\end{array}$ & $\begin{array}{c}\text { Not } \\
\text { introduced at } \\
\text { that time }\end{array}$ & - & $\begin{array}{c}\text { Not } \\
\text { introduced }\end{array}$ & $\begin{array}{c}\text { Not } \\
\text { introduced }\end{array}$ & $\begin{array}{c}\text { not use } \\
\text { significantly } \\
\text { as training } \\
\text { tool }\end{array}$ & $\begin{array}{l}\text { Continuously } \\
\text { increasing its } \\
\text { popularity as } \\
\text { training tool. }\end{array}$ \\
\hline 5 & $\begin{array}{l}\text { Simulation } \\
\text { exercise }\end{array}$ & $\begin{array}{l}\text { Not } \\
\text { introduced } \\
\text { that time }\end{array}$ & - & $\begin{array}{l}\text { Not used as } \\
\text { training tool }\end{array}$ & $\begin{array}{c}\text { Not } \\
\text { available }\end{array}$ & $\begin{array}{l}\text { Not used as } \\
\text { costly }\end{array}$ & $\begin{array}{l}\text { Did not focus } \\
\text { on it }\end{array}$ \\
\hline 6 & $\begin{array}{l}\text { Vestibule } \\
\text { training }\end{array}$ & $\begin{array}{c}\text { Not } \\
\text { introduced }\end{array}$ & - & $\begin{array}{c}\text { Not } \\
\text { introduced }\end{array}$ & $\begin{array}{c}\text { Not } \\
\text { introduced }\end{array}$ & $\begin{array}{c}\text { learn the } \\
\text { operating } \\
\text { process of } \\
\text { new software, } \\
\text { equipments } \\
\text { (length } 7-15 \\
\text { days) }\end{array}$ & Less focused \\
\hline 7 & $\begin{array}{l}\text { Programmed } \\
\text { instruction }\end{array}$ & $\begin{array}{c}\text { Used as } \\
\text { training } \\
\text { instruction } \\
\text { not as a tool }\end{array}$ & - & $\begin{array}{l}\text { Instruction } \\
\text { schedule in } \\
\text { training } \\
\text { session. }\end{array}$ & $\begin{array}{c}\text { used as } \\
\text { training } \\
\text { schedule at } \\
\text { that time }\end{array}$ & $\begin{array}{l}\text { Training } \\
\text { instruction } \\
\text { schedule }\end{array}$ & $\begin{array}{l}\text { Done as a } \\
\text { training } \\
\text { schedule }\end{array}$ \\
\hline 8 & Job rotation & $\begin{array}{c}\text { Formally } \\
\text { each } \\
\text { department } \\
\text { should rotate } \\
\text { job in each } 3 \\
\text { months }\end{array}$ & - & $\begin{array}{l}\text { follow it but } \\
\text { not strictly }\end{array}$ & $\begin{array}{l}\text { Basically } \\
\text { done this } \\
\text { with the } \\
\text { provisional } \\
\text { officer or } \\
\text { MTO, }\end{array}$ & $\begin{array}{l}\text { Follow it but } \\
\text { moderately }\end{array}$ & $\begin{array}{l}\text { For MTO and } \\
\text { PO, rotate after } \\
1 \text { year or in } 6 \\
\text { month around } \\
\text { the departments. }\end{array}$ \\
\hline 9 & Role playing & Not applied & - & Not applied & Not applied & Not applied & Not applied \\
\hline
\end{tabular}

Training Development Criteria: All banks provide training (class lecture) to their employees through their training academy. In private banks Managing Director decides who should get training. The popularity of Audiovisual techniques have increased in private banks between 1991 to 2010, before that it was not introduced in the banks as stated in Table 4. Vestibule training was introduced in public banks in 2000. Due to an acute change in the technical side and the effectiveness of this tool it is used as additional training tool under the experts as workshop for $7-15$ days among the group of employees from different branches who need to learn the new operating process or new thing to perform better. It is similar to the Simulation Exercise but it is yet not used in private banks as it lacks attention toward their employee's training need and cost. In public bank, Job rotation is mandatory for all newly recruited during 3 months of recruiting around the departments but it's followed mildly whereas in private bank, it is conducted only with the provisional officer or MTO. There is no clear concept about the programmed instructions; it mainly refers to the training schedule in the private and public bank.

TABLE 5: d) Promotion Criteria:

\begin{tabular}{|c|c|c|c|c|c|c|}
\hline \multirow{3}{*}{ Promotion criteria } & \multirow{2}{*}{\multicolumn{2}{|c|}{$\begin{array}{c}\text { 1971-1980 } \\
\text { Overall Industries }\end{array}$}} & \multirow{2}{*}{\multicolumn{2}{|c|}{$\begin{array}{c}1981-1990 \\
\text { Overall Industries }\end{array}$}} & \multirow{2}{*}{\multicolumn{2}{|c|}{$\begin{array}{c}\text { 1991-2010 } \\
\text { Overall Industries }\end{array}$}} \\
\hline & & & & & & \\
\hline & Public & Private & Public & Private & Public & Private \\
\hline $\begin{array}{l}\text { 1. Length of } \\
\text { service }\end{array}$ & $\begin{array}{l}3 \text { years for } \\
\text { graduated, } 5 \\
\text { year for non } \\
\text { graduated } \\
\text { employees } \\
\text { entitled for } \\
\text { promotion }\end{array}$ & - & $\begin{array}{c}3 \text { years for } \\
\text { graduate and } 5 \\
\text { years for } \\
\text { undergraduate. }\end{array}$ & $\begin{array}{c}\text { After each } 3 \\
\text { years, entitled } \\
\text { to get } \\
\text { promotion } \\
\text { along with } \\
\text { other criteria }\end{array}$ & $\begin{array}{l}\text { After each } 3 \\
\text { years, } \\
\text { graduated } \\
\text { employee, } 5 \\
\text { year non } \\
\text { graduated } \\
\text { employees }\end{array}$ & $\begin{array}{c}\text { After } 3 \text { years, } \\
\text { entitled to get } \\
\text { promotion }\end{array}$ \\
\hline
\end{tabular}




\begin{tabular}{|c|c|c|c|c|c|c|c|}
\hline 2 & $\begin{array}{l}\text { Recommendation } \\
\text { by direct } \\
\text { supervisor }\end{array}$ & $\begin{array}{l}\text { Along with } \\
\text { other criteria, } \\
\text { manager's } \\
\text { recommendatio } \\
\text { n influences to } \\
\text { some extent. }\end{array}$ & - & $\begin{array}{l}\text { Managers try } \\
\text { to give better } \\
\text { feedback or } \\
\text { recommend } \\
\text { the employee } \\
\text { to top } \\
\text { management }\end{array}$ & $\begin{array}{l}\text { Manager } \\
\text { recommendati } \\
\text { ons influence } \\
\text { most the top } \\
\text { management's } \\
\text { decision in } \\
\text { this sector. }\end{array}$ & $\begin{array}{l}\text { Most of the } \\
\text { times they } \\
\text { recommend } \\
\text { better, hence } \\
\text { have great } \\
\text { influence. }\end{array}$ & $\begin{array}{l}\text { Manager's } \\
\text { recommendati } \\
\text { on influences } \\
\text { promotion } \\
\text { most. }\end{array}$ \\
\hline 3 & Based on merit & $\begin{array}{l}\text { Highly } \\
\text { influential } \\
\text { factor }\end{array}$ & - & $\begin{array}{l}\text { Highly } \\
\text { influential } \\
\text { factor }\end{array}$ & $\begin{array}{l}\text { Influence } \\
\text { promotional } \\
\text { decision. }\end{array}$ & $\begin{array}{l}\text { Highly } \\
\text { influential } \\
\text { factor }\end{array}$ & $\begin{array}{l}\text { Highly } \\
\text { influential } \\
\text { factor }\end{array}$ \\
\hline 4 & $\begin{array}{l}\text { Right attitudes } \\
\text { and values }\end{array}$ & $\begin{array}{c}\text { Yes have } \\
\text { impact on } \\
\text { promotional } \\
\text { decision }\end{array}$ & - & $\begin{array}{l}\text { have impact } \\
\text { on } \\
\text { promotional }\end{array}$ & $\begin{array}{l}\text { Not consider } \\
\text { this criteria } \\
\text { much at that } \\
\text { time }\end{array}$ & $\begin{array}{c}\text { have impact on } \\
\text { promotional }\end{array}$ & Very Rare \\
\hline 5 & $\begin{array}{l}\text { Knowledge and } \\
\text { skill }\end{array}$ & $\begin{array}{l}\text { Highly Matter } \\
\text { in promotional } \\
\text { decision }\end{array}$ & - & $\begin{array}{l}\text { Performance } \\
\text { and skill have } \\
\text { impact on } \\
\text { promotional } \\
\text { decision. }\end{array}$ & $\begin{array}{l}\text { Have Impact } \\
\text { to some extent } \\
\text { along with } \\
\text { other factors }\end{array}$ & $\begin{array}{l}\text { sometime Best } \\
\text { performed } \\
\text { employee } \\
\text { deprived from } \\
\text { getting } \\
\text { promotion due } \\
\text { to reference }\end{array}$ & $\begin{array}{l}\text { Have Impact to } \\
\text { some extent }\end{array}$ \\
\hline 6 & Record of training & $\begin{array}{l}\text { Taken into } \\
\text { account the test } \\
\text { result of the } \\
\text { training at } \\
\text { certain } \%\end{array}$ & - & $\begin{array}{l}\text { have certain } \\
\text { impact on } \\
\text { promotional } \\
\text { decision }\end{array}$ & $\begin{array}{l}\text { Did not } \\
\text { consider its } \\
\text { impact on } \\
\text { promotional } \\
\text { decision }\end{array}$ & $\begin{array}{c}\text { Till now have } \\
\text { certain impact } \\
\text { on promotional } \\
\text { decision }\end{array}$ & $\begin{array}{l}\text { Did not have } \\
\text { any impact on } \\
\text { promotional } \\
\text { decision }\end{array}$ \\
\hline 7 & $\begin{array}{l}\text { Performance } \\
\text { evaluation }\end{array}$ & $\begin{array}{l}\text { Highly } \\
\text { influence the } \\
\text { promotion } \\
\text { decision }\end{array}$ & - & $\begin{array}{l}\text { Management } \\
\text { decision } \\
\text { influenced by } \\
\text { it }\end{array}$ & $\begin{array}{l}\text { Highly } \\
\text { followed }\end{array}$ & $\begin{array}{l}\text { Highly } \\
\text { followed }\end{array}$ & $\begin{array}{c}\text { Highly } \\
\text { influence the } \\
\text { decision }\end{array}$ \\
\hline 8 & Party membership & NA & - & NA & NA & NA & NA \\
\hline
\end{tabular}

Promotion Criteria: Table 5 demonstrate that public banks evaluates each performance criteria like -whether the ACR (Annual Confidential Report) marks contains 50-65\% in the promotion, KSA (knowledge, skills and abilities), educational degree, competency to handle customer or performance or training period or behavior and supervisors observation during promotion. Public banks practice performance based promotion. It motivates the employee in public sector since supervisors recommendation is not everything. Unlike Public Banks, Private Banks emphasizes mainly the performance, merit. KSA, ACR marking supervisor, chairmen, manager's recommendation and also the central bank influenced promotion during $1985-2010$, but they underestimated training period performance. In both banks there are close competition of employees for getting promotion \& reward but in most cases reference or influence of less skilled employees deprive the deserving one or delay the promotion. There is a facility called "time scale pay", which was introduced by the public banks in 2000 , to compensate those deserving employees who could not get promotion on time by giving reward or extra pay as promotion increment.

TABLE 6: e) Performance Appraisal Criteria:

\begin{tabular}{|c|c|c|c|c|c|c|c|}
\hline \multirow{4}{*}{\multicolumn{2}{|c|}{$\begin{array}{c}\text { Performance appraisal } \\
\text { criteria }\end{array}$}} & \multirow{3}{*}{\multicolumn{2}{|c|}{$\begin{array}{c}\text { 1971-1980 } \\
\text { Overall Industries }\end{array}$}} & \multirow{3}{*}{\multicolumn{2}{|c|}{$\begin{array}{c}\text { 1981-1990 } \\
\text { Overall Industries }\end{array}$}} & \multirow{3}{*}{\multicolumn{2}{|c|}{$\begin{array}{c}\text { 1991-2010 } \\
\text { Overall Industries }\end{array}$}} \\
\hline & & & & & & & \\
\hline & & & & & & & \\
\hline & & Public & Pvt. & Public & Private & Public & Private \\
\hline & 1.Essay Appraisal & Not followed & - & Not followed & $\begin{array}{c}\text { Not } \\
\text { followed }\end{array}$ & Not followed & $\begin{array}{c}\text { Not } \\
\text { performed }\end{array}$ \\
\hline 2 & Graphic Rating Scale & $\begin{array}{l}\text { Some portion } \\
\text { of the } \\
\text { appraisal used } \\
\text { this } \\
\text { techniques }\end{array}$ & - & $\begin{array}{l}\text { Some portion of } \\
\text { the appraisal } \\
\text { used this } \\
\text { techniques }\end{array}$ & $\begin{array}{l}\text { Not } \\
\text { followed }\end{array}$ & $\begin{array}{l}\text { Some portion } \\
\text { of the } \\
\text { appraisal used } \\
\text { this } \\
\text { techniques }\end{array}$ & $\begin{array}{l}\text { Not } \\
\text { performed }\end{array}$ \\
\hline 3 & $\begin{array}{c}\text { Behaviorally Anchored } \\
\text { Rating Scale }\end{array}$ & Not followed & - & Not followed & $\begin{array}{c}\text { Not } \\
\text { followed }\end{array}$ & Not followed & $\begin{array}{c}\text { Not } \\
\text { performed }\end{array}$ \\
\hline 4 & Field Review & $\begin{array}{c}\text { Not } \\
\text { considered }\end{array}$ & - & Not followed & $\begin{array}{c}\text { Not } \\
\text { followed }\end{array}$ & Not followed & $\begin{array}{c}\text { Not } \\
\text { Performed }\end{array}$ \\
\hline 5 & $\begin{array}{l}\text { Simple Ranking / } \\
\text { Rating }\end{array}$ & $\begin{array}{l}\text { Mostly used } \\
\text { this rating }\end{array}$ & - & $\begin{array}{l}\text { Mostly used this } \\
\text { rating }\end{array}$ & $\begin{array}{l}\text { Mostly } \\
\text { used this } \\
\text { rating }\end{array}$ & $\begin{array}{l}\text { Mostly used } \\
\text { this rating }\end{array}$ & $\begin{array}{c}\text { Follow this } \\
\text { technique } \\
\text { most }\end{array}$ \\
\hline 6 & Forced Choice Rating & $\begin{array}{c}\text { Not } \\
\text { performed }\end{array}$ & - & Not performed & $\begin{array}{c}\text { Not } \\
\text { performed }\end{array}$ & $\begin{array}{c}\text { Not } \\
\text { performed }\end{array}$ & $\begin{array}{l}\text { Not } \\
\text { performed }\end{array}$ \\
\hline 7 & $\begin{array}{l}\text { Critical Incident } \\
\text { Appraisal }\end{array}$ & $\begin{array}{l}\text { Manager } \\
\text { sometime } \\
\text { keep track of } \\
\text { it while }\end{array}$ & - & $\begin{array}{l}\text { Manager } \\
\text { sometime keep } \\
\text { track of it while } \\
\text { promoting }\end{array}$ & $\begin{array}{c}\text { not } \\
\text { widely } \\
\text { used as } \\
\text { performan }\end{array}$ & $\begin{array}{c}\text { sometime } \\
\text { keep track of } \\
\text { it }\end{array}$ & $\begin{array}{l}\text { Manager } \\
\text { sometime } \\
\text { keep track of } \\
\text { it while }\end{array}$ \\
\hline
\end{tabular}




\begin{tabular}{|c|c|c|c|c|c|c|c|}
\hline & & $\begin{array}{l}\text { promoting } \\
\text { employees }\end{array}$ & & employees & $\begin{array}{l}\text { ce was the } \\
\text { main } \\
\text { factor }\end{array}$ & & $\begin{array}{l}\text { promoting } \\
\text { employees }\end{array}$ \\
\hline 8 & $\begin{array}{l}\text { Management by } \\
\text { Objective }\end{array}$ & $\begin{array}{l}\text { Did not } \\
\text { applied }\end{array}$ & - & Did not applied & $\begin{array}{l}\text { Did not } \\
\text { applied }\end{array}$ & $\begin{array}{l}\text { Did not } \\
\text { applied }\end{array}$ & $\begin{array}{l}\text { Did not } \\
\text { applied }\end{array}$ \\
\hline 9 & $\begin{array}{c}\text { ACR (Annual } \\
\text { Confidential Report) }\end{array}$ & $\begin{array}{l}\text { Major } \\
\text { component for } \\
\text { performance } \\
\text { appraisal }\end{array}$ & - & $\begin{array}{c}\text { Major } \\
\text { component for } \\
\text { performance } \\
\text { appraisal }\end{array}$ & $\begin{array}{c}\text { Major } \\
\text { componen } \\
t \text { for } \\
\text { performan } \\
\text { ce } \\
\text { appraisal }\end{array}$ & $\begin{array}{l}\text { Major } \\
\text { component for } \\
\text { performance } \\
\text { appraisal }\end{array}$ & $\begin{array}{l}\text { Major } \\
\text { component of } \\
\text { performance } \\
\text { appraisal }\end{array}$ \\
\hline
\end{tabular}

Performance Appraisal Criteria: Both public and private banks have not been following any specific technique in performance appraisal from $1972-2010$, as stated in Table 6 . They combined all the techniques to get the best appraisal or evaluation based on - ACR (Annual Confidential Report) marks, Field Review, Critical Incident Appraisal, Simple Rating etc. All the banks rate the areas of appraisal based on the behavior, target fulfilling or in terms of services provided or comment and then provide judgment or opinion for it or rate according to $1-10$ scale observing the employees attitude, skill, work understanding capability \& behavior. Some criteria are evaluated based on the total marks obtained in the ranking. Final evaluation is done by combining all the marks or score; but both types of banks prioritize the reference of the top level management or the supervisor's comments.

TABLE 7: f) Compensation Criteria:

\begin{tabular}{|c|c|c|c|c|c|c|c|}
\hline & \multirow{4}{*}{$\begin{array}{c}\text { Compensation } \\
\text { criteria }\end{array}$} & \multirow{3}{*}{\multicolumn{2}{|c|}{$\begin{array}{c}\text { 1971-1980 } \\
\text { Overall Industries }\end{array}$}} & \multirow{3}{*}{\multicolumn{2}{|c|}{$\begin{array}{c}1981-1990 \\
\text { Overall Industries }\end{array}$}} & \multirow{3}{*}{\multicolumn{2}{|c|}{$\begin{array}{c}\text { 1991-2010 } \\
\text { Overall Industries }\end{array}$}} \\
\hline & & & & & & & \\
\hline & & & & & & & \\
\hline & & \multirow[b]{2}{*}{$\begin{array}{c}\text { Public } \\
\text { Overall } \\
\text { performance } \\
\text { is the major } \\
\text { factor while } \\
\text { allocating } \\
\text { compensatio } \\
n\end{array}$} & \multirow[t]{2}{*}{ Pvt. } & Public & Private & Public & Private \\
\hline & $\begin{array}{l}\text { 1.Employees' } \\
\text { performance }\end{array}$ & & & $\begin{array}{l}\text { Final result } \\
\text { played an } \\
\text { important role at } \\
\text { that period. }\end{array}$ & $\begin{array}{l}\text { Main } \\
\text { influencing } \\
\text { factor while } \\
\text { giving the } \\
\text { compensation }\end{array}$ & $\begin{array}{l}\text { Service provided } \\
\text { or overall } \\
\text { performance } \\
\text { played the vital } \\
\text { role }\end{array}$ & $\begin{array}{l}\text { Target fill up etc., } \\
\text { overall } \\
\text { performance is the } \\
\text { key influencing } \\
\text { factor }\end{array}$ \\
\hline 2 & Skill-based pay & $\begin{array}{c}\text { Yes payment } \\
\text { also } \\
\text { influenced } \\
\text { by this } \\
\text { factor. }\end{array}$ & - & $\begin{array}{l}\text { Most influential } \\
\text { factor while } \\
\text { deciding } \\
\text { compensation }\end{array}$ & $\begin{array}{l}\text { Compensation } \\
\text { decision some } \\
\text { extent } \\
\text { influenced } \\
\text { based on skill }\end{array}$ & $\begin{array}{l}\text { Compensation or } \\
\text { reward also is } \\
\text { provided based on } \\
\text { the KSA, merit }\end{array}$ & $\begin{array}{l}\text { Refer to pay for } \\
\text { performance, some } \\
\text { extent influence the } \\
\text { compensation. }\end{array}$ \\
\hline 3 & $\begin{array}{l}\text { Sweet-will of the } \\
\text { employer }\end{array}$ & $\begin{array}{l}\text { Have impact } \\
\text { on final } \\
\text { evaluation } \& \\
\text { compensatio } \\
\text { n decision. }\end{array}$ & - & $\begin{array}{l}\text { Influence to } \\
\text { some extent }\end{array}$ & $\begin{array}{l}\text { N/A except } \\
\text { some special } \\
\text { case }\end{array}$ & $\begin{array}{l}\text { Influence } \\
\text { sometime the final } \\
\text { rating of } \\
\text { employees }\end{array}$ & $\begin{array}{l}\text { N/A except some } \\
\text { special case }\end{array}$ \\
\hline 4 & $\begin{array}{l}\text { Pay structure of } \\
\text { the government } \\
\text { of Bangladesh }\end{array}$ & $\begin{array}{c}\text { Follow } \\
\text { government } \\
\text { fixed pay } \\
\text { structure }\end{array}$ & - & $\begin{array}{c}\text { Follow } \\
\text { government fixed } \\
\text { pay structure }\end{array}$ & $\begin{array}{l}\text { Not applicable } \\
\text { as private bank }\end{array}$ & $\begin{array}{c}\text { Follow } \\
\text { government fixed } \\
\text { pay structure }\end{array}$ & $\begin{array}{c}\text { Not applicable as } \\
\text { private bank }\end{array}$ \\
\hline 5 & $\begin{array}{l}\text { Pay structure of } \\
\text { self organizations } \\
\text { in the industry }\end{array}$ & $\begin{array}{c}\text { Not } \\
\text { applicable as } \\
\text { public bank }\end{array}$ & - & $\begin{array}{l}\text { Not applicable as } \\
\text { public bank }\end{array}$ & $\begin{array}{l}\text { Own or self pay } \\
\text { structure }\end{array}$ & $\begin{array}{c}\text { Not applicable as } \\
\text { public bank }\end{array}$ & $\begin{array}{l}\text { Own or self pay } \\
\text { structure }\end{array}$ \\
\hline 6 & $\begin{array}{c}\text { compensation for } \\
\text { outstay benefit }\end{array}$ & $\begin{array}{l}\text { No extra } \\
\text { facility } \\
\text { provided, } \\
\text { some cases } \\
\text { gave less } \\
\text { compensatio } \\
\text { n while } \\
\text { outstaying }\end{array}$ & - & $\begin{array}{c}\text { No facility } \\
\text { provided, } \\
\text { sometime give } \\
\text { less } \\
\text { compensation for } \\
\text { those who } \\
\text { outstay }\end{array}$ & $\begin{array}{c}\text { No facility } \\
\text { provided, gave } \\
\text { less } \\
\text { compensation }\end{array}$ & $\begin{array}{l}\text { No facility } \\
\text { provided, give less } \\
\text { compensation }\end{array}$ & $\begin{array}{l}\text { No facility } \\
\text { provided, give less } \\
\text { compensation }\end{array}$ \\
\hline
\end{tabular}

Compensation Criteria: During 1971 - 2010, as Table 7 shows, both types of banks focused on the employee's overall performance especially for setting compensation. Compensation is given based on the performance, competency in handling customer, target fill up etc. Both the banks give priority to skill- based pay which motivates the employees to some level and there is a best performer reward in both banks. Public banks follow government pay structure whereas private focuses on self organized pay structure, both industries provide no facility or extra benefit for outstay, some cases give low compensation while outstaying. Public banks provide $40 \%$ house rent instead of $50 \%$ and private banks provide $4000-5000$ BDT for food, accommodation and transportation cost for outstaying. 
TABLE 8: g) Benefits \& Services Criteria:

\begin{tabular}{|c|c|c|c|c|c|c|c|}
\hline \multirow{4}{*}{\multicolumn{2}{|c|}{$\begin{array}{c}\text { Benefits \& } \\
\text { services }\end{array}$}} & & & \multirow{3}{*}{\multicolumn{2}{|c|}{$\begin{array}{c}\text { 1981-1990 } \\
\text { Overall Industries }\end{array}$}} & \multirow{3}{*}{\multicolumn{2}{|c|}{$\begin{array}{c}\text { 1991-2010 } \\
\text { Overall Industries }\end{array}$}} \\
\hline & & \multirow{2}{*}{\multicolumn{2}{|c|}{$\begin{array}{c}1971-1980 \\
\text { Overall Industries }\end{array}$}} & & & & \\
\hline & & & & & & & \\
\hline & & Public & Pvt. & Public & Private & Public & Private \\
\hline & $\begin{array}{c}\text { 1. House } \\
\text { rent } \\
\text { allowance/R } \\
\text { ent free } \\
\text { accommodat } \\
\text { ion }\end{array}$ & $\begin{array}{c}\text { Give this based } \\
\text { on government } \\
\text { pay structure }\end{array}$ & & $\begin{array}{l}\text { Give this based } \\
\text { on government } \\
\text { pay structure }\end{array}$ & $\begin{array}{c}\text { Give } \\
\text { allowances } \\
\text { (BDT5000) }\end{array}$ & $\begin{array}{l}\text { Give this } \\
\text { based on } \\
\text { overnment } \\
\text { pay structure }\end{array}$ & $\begin{array}{l}\text { Position basis house } \\
\text { allowances given }\end{array}$ \\
\hline 2 & $\begin{array}{c}\text { Car/ } \\
\text { Conveyance } \\
\text { allowance }\end{array}$ & $\begin{array}{l}\text { Car allowance is } \\
\text { given to junior } \\
\text { \& car facility is } \\
\text { for top level } \\
\text { management }\end{array}$ & & $\begin{array}{l}\text { (BDT } 25 \text { for bus } \\
\text { rent ) for lower } \\
\text { employees and } \\
\text { car facility, loan } \\
\text { is for top level } \\
\text { employee }\end{array}$ & $\begin{array}{c}\text { Car for top } \\
\text { level executive } \\
\text { only, no } \\
\text { facility for } \\
\text { junior level } \\
\text { employees. Pay } \\
\text { only in holiday } \\
\text { if work }\end{array}$ & $\begin{array}{c}\text { BDT } 40 \text { for } \\
\text { bus rent for } \\
\text { lower level } \\
\text { employees } \\
\text { and car and } \\
\text { loan is for top } \\
\text { level } \\
\text { management }\end{array}$ & $\begin{array}{c}\text { For top level executive } \\
\text { only (VP to MD), gave } \\
\text { BDT150 as } \\
\text { transportation cost for } \\
\text { lower or junior level } \\
\text { employees only } \\
\text { Saturday }\end{array}$ \\
\hline 3 & $\begin{array}{l}\text { Medical } \\
\text { allowance }\end{array}$ & $\begin{array}{l}\text { Allowance is } \\
\text { provided } \\
\text { according to the } \\
\text { overnment } \\
\text { rated percentage }\end{array}$ & - & $\begin{array}{l}\text { Allowance is } \\
\text { provided } \\
\text { according to the } \\
\text { government } \\
\text { rated percentage }\end{array}$ & $\begin{array}{c}\text { Gave } \\
\text { allowances } \\
\text { along with } \\
\text { admission } \\
\text { facility in the } \\
\text { sister concerns } \\
\text { organization }\end{array}$ & $\begin{array}{l}\text { According to } \\
\text { overnment } \\
\text { scale, Bank } \\
\text { now provided } \\
\text { BDT700 as } \\
\text { medical } \\
\text { allowances. }\end{array}$ & $\begin{array}{l}\text { Allowance is provided } \\
\text { according to the } \\
\text { position (currently } \\
\text { BDT } 5000-10000)\end{array}$ \\
\hline 4 & $\begin{array}{l}\text { Telephone } \\
\text { allowance }\end{array}$ & $\begin{array}{c}\text { No allowance } \\
\text { provided }\end{array}$ & & $\begin{array}{c}\text { No allowance } \\
\text { provided }\end{array}$ & $\begin{array}{l}\text { No facility } \\
\text { provided }\end{array}$ & $\begin{array}{l}\text { For top level } \\
\text { management, } \\
\text { give fixed } \\
\text { allowance } \\
\text { (BDT } 200- \\
400 \text { for } \\
\text { mobile during } \\
\text { the year 2007 } \\
-2008 \text { ) }\end{array}$ & Not provided \\
\hline 5 & $\begin{array}{l}\text { Entertainme } \\
\text { nt allowance }\end{array}$ & $\begin{array}{c}\text { No facility } \\
\text { provided, till } \\
\text { banks arrange } \\
\text { this by their own } \\
\text { fund }\end{array}$ & - & $\begin{array}{c}\text { No facility } \\
\text { provided, till } \\
\text { banks arrange } \\
\text { this by their own } \\
\text { fund }\end{array}$ & $\begin{array}{c}\text { Yes annual } \\
\text { picnic was held } \\
\text { but not } \\
\text { provided } \\
\text { allowances on } \\
\text { individual } \\
\text { employees } \\
\text { account }\end{array}$ & $\begin{array}{l}\text { Banks arrange } \\
\text { this as a form } \\
\text { of picnic by } \\
\text { their own, no } \\
\text { allowances is } \\
\text { been } \\
\text { provided. }\end{array}$ & $\begin{array}{l}\text { Annual picnic was held } \\
\text { but not provided } \\
\text { allowances on } \\
\text { individual employees } \\
\text { account }\end{array}$ \\
\hline 6 & Bonus & $\begin{array}{l}2 \text { festival bonus } \\
\text { and } 2 \text { and half } \\
\text { bonus }\end{array}$ & & $\begin{array}{l}2 \text { festival bonus } \\
\text { and } 2 \text { and half } \\
\text { bonus, some } \\
\text { time got total } 5 \\
\text { bonus }\end{array}$ & $\begin{array}{l}1 \text { bonus for } \\
\text { either festival. }\end{array}$ & $\begin{array}{l}2 \text { festival } \\
\text { bonus and } 2 \\
\text { and half } \\
\text { bonus, some } \\
\text { time got total } \\
5 \text { bonus }\end{array}$ & $\begin{array}{l}1 \text { bonus for either } \\
\text { festival }\end{array}$ \\
\hline 7 & $\begin{array}{l}\text { Leave with } \\
\text { pay }\end{array}$ & $\begin{array}{l}\text { Give paid leave, } \\
\text { need to inform } \\
\text { at least } 1 \text { month } \\
\text { earlier before } \\
\text { quieting }\end{array}$ & & $\begin{array}{l}\text { Give paid leave, } \\
\text { minimum } 1 \\
\text { month prior } \\
\text { notice of leaving }\end{array}$ & $\begin{array}{l}\text { Give paid } \\
\text { leave, } \\
\text { minimum } 2 \\
\text { month prior } \\
\text { notice of } \\
\text { leaving. }\end{array}$ & $\begin{array}{l}\text { Give paid } \\
\text { leave, } \\
\text { minimum } 1 \\
\text { month prior } \\
\text { notice of } \\
\text { leaving. }\end{array}$ & 60days leave with pay \\
\hline 8 & $\begin{array}{l}\text { Recreation } \\
\text { allowance }\end{array}$ & $\begin{array}{l}\text { No facility } \\
\text { provided }\end{array}$ & & $\begin{array}{l}\text { No facility } \\
\text { provided }\end{array}$ & $\begin{array}{l}\text { No facility } \\
\text { provided }\end{array}$ & $\begin{array}{l}\text { No facility } \\
\text { provided }\end{array}$ & $\begin{array}{l}\text { Some case give } 15 \\
\text { days' vacation in a year } \\
\text { with pay }\end{array}$ \\
\hline 9 & $\begin{array}{l}\text { Children's } \\
\text { education } \\
\text { facility }\end{array}$ & $\begin{array}{c}\text { Gave according } \\
\text { to the } \\
\text { overnment } \\
\text { allotted } \\
\text { percentage }\end{array}$ & & $\begin{array}{l}\text { Gave according } \\
\text { to the } \\
\text { government } \\
\text { allotted } \\
\text { percentage }\end{array}$ & $\begin{array}{l}\text { No facility } \\
\text { provided }\end{array}$ & $\begin{array}{l}\text { Gave } \\
\text { according to } \\
\text { the } \\
\text { overnment } \\
\text { allotted } \\
\text { percentage } \\
\text { now BDT } 300 \\
\text { is given. }\end{array}$ & No facility provided \\
\hline 10 & $\begin{array}{l}\text { Home } \\
\text { furnishing } \\
\text { allowance }\end{array}$ & Not provided & & Not provided & $\begin{array}{l}\text { No facility } \\
\text { provided }\end{array}$ & Not provided & No facility provided \\
\hline 11 & $\begin{array}{l}\text { Electricity } \\
\text { and Gas } \\
\text { expenses }\end{array}$ & Not provided & - & Not provided & $\begin{array}{l}\text { No facility } \\
\text { provided }\end{array}$ & Not provided & No facility provided \\
\hline 12 & $\begin{array}{l}\text { Company } \\
\text { products at }\end{array}$ & No specific rule & - & No specific rule & $\begin{array}{c}\text { No facility } \\
\text { provided (e.g. }\end{array}$ & $\begin{array}{l}\text { No specific } \\
\text { rule but can }\end{array}$ & No facility provided \\
\hline
\end{tabular}




\begin{tabular}{|c|c|c|c|c|c|c|}
\hline & $\begin{array}{l}\text { subsidized } \\
\text { price }\end{array}$ & & & $\begin{array}{l}\text { loan interest } \\
\text { rate is higher } \\
\text { than from other } \\
\text { public bank for } \\
\text { employees) }\end{array}$ & $\begin{array}{l}\text { take loan but } \\
\text { at bank's } \\
\text { normal } \\
\text { interest rate }\end{array}$ & \\
\hline 13 & Sick leave & $\begin{array}{l}\text { Paid leave, fixed } \\
\text { amount is paid } \\
\text { BDT } 700 .\end{array}$ & $\begin{array}{c}\text { Paid leave, fixed } \\
\text { amount is paid } \\
\text { BDT } 700 .\end{array}$ & $\begin{array}{l}\text { Deduct } \\
\text { BDT150 if } \\
\text { leave in } \\
\text { Sunday \& } \\
\text { Thursday, } \\
\text { other day was } \\
\text { considered as } \\
\text { casual leave } \\
\text { with pay. }\end{array}$ & $\begin{array}{c}\text { Paid leave, } \\
\text { fixed amount } \\
\text { BDT } 700 \text { is } \\
\text { paid }\end{array}$ & $\begin{array}{c}\text { Yes gave paid sick } \\
\text { leave }\end{array}$ \\
\hline 14 & $\begin{array}{l}\text { Maternity } \\
\text { leave }\end{array}$ & $\begin{array}{l}40 \text { to } 42 \text { days } \\
\text { paid leave }\end{array}$ & $\begin{array}{l}3 \text { month with } \\
\text { paid leave for } \\
\text { those who are } \\
\text { employed for } \\
\text { over } 2 \text { year. }\end{array}$ & $\begin{array}{l}3 \text { month paid } \\
\text { leave for those } \\
\text { who are } \\
\text { employed for } \\
\text { over } 2 \text { year. }\end{array}$ & $\begin{array}{l}6 \text { month paid } \\
\text { leave for } \\
\text { those who are } \\
\text { employed for } \\
\text { over } 2 \text { year. }\end{array}$ & $\begin{array}{l}6 \text { months with pay for } \\
\text { those who are } \\
\text { employed for over } 2 \\
\text { year. }\end{array}$ \\
\hline 15 & $\begin{array}{l}\text { Paternity } \\
\text { leave }\end{array}$ & $\begin{array}{c}\text { Did not give this } \\
\text { leave }\end{array}$ & $\begin{array}{c}\text { Did not give this } \\
\text { leave }\end{array}$ & $\begin{array}{l}\text { Did not give } \\
\text { this leave }\end{array}$ & $\begin{array}{l}\text { Did not give } \\
\text { this leave }\end{array}$ & Did not give this leave \\
\hline 16 & $\begin{array}{l}\text { Marriage } \\
\text { leave }\end{array}$ & $\begin{array}{l}\text { Did not give this } \\
\text { leave, need to } \\
\text { take casual leave } \\
\text { (CL) }\end{array}$ & $\begin{array}{c}\text { Did not give this } \\
\text { leave need to } \\
\text { take casual leave }\end{array}$ & $\begin{array}{c}\text { Did not give } \\
\text { this leave need } \\
\text { to take casual } \\
\text { leave }\end{array}$ & $\begin{array}{l}\text { Did not give } \\
\text { this leave } \\
\text { need to take } \\
\text { casual leave }\end{array}$ & $\begin{array}{c}\text { Did not give this leave } \\
\text { need to take casual } \\
\text { leave }\end{array}$ \\
\hline 17 & $\begin{array}{l}\text { Compensati } \\
\text { on for short- } \\
\text { term } \\
\text { disability }\end{array}$ & $\begin{array}{l}\text { No specific fund } \\
\text { for it, bank gave } \\
\text { from the general } \\
\text { fund. }\end{array}$ & $\begin{array}{c}\text { No specific fund } \\
\text { for it, bank will } \\
\text { bear the cost if } \\
\text { disability is } \\
\text { happen while } \\
\text { working using } \\
\text { general fund. }\end{array}$ & $\begin{array}{l}\text { No specific } \\
\text { leave for it, } \\
\text { need to take } \\
\text { sick leave and } \\
\text { medical facility } \\
\text { is provided at } \\
\text { certain limit }\end{array}$ & $\begin{array}{l}\text { No specific } \\
\text { fund for it, } \\
\text { bear the cost } \\
\text { if disability is } \\
\text { happen while } \\
\text { working from } \\
\text { the general } \\
\text { fund. }\end{array}$ & $\begin{array}{l}\text { No specific leave for it, } \\
\text { need to take sick leave } \\
\text { and medical facility is } \\
\text { provided at certain } \\
\text { limit }\end{array}$ \\
\hline 18 & $\begin{array}{l}\text { Compensati } \\
\text { on for long- } \\
\text { term } \\
\text { disability }\end{array}$ & $\begin{array}{l}\text { Bank maintained } \\
\text { the cost, if } \\
\text { employee can't } \\
\text { be physically fit, } \\
\text { forced } \\
\text { retirement was } \\
\text { initiated }\end{array}$ & $\begin{array}{c}\text { Bank paid if this } \\
\text { happen while } \\
\text { working, if } \\
\text { employee can't } \\
\text { be physically fit, } \\
\text { forced } \\
\text { retirement was } \\
\text { initiated }\end{array}$ & $\begin{array}{l}\text { Force to go for } \\
\text { retirement }\end{array}$ & $\begin{array}{l}\text { Bank paid if } \\
\text { this happen } \\
\text { while } \\
\text { working, } \\
\text { maintained } \\
\text { the cost, if } \\
\text { employee } \\
\text { can't be fit, } \\
\text { forced } \\
\text { retirement }\end{array}$ & $\begin{array}{l}\text { Force to go for } \\
\text { retirement }\end{array}$ \\
\hline
\end{tabular}

Benefits \& Services Criteria: Benefits and services vary from bank to bank due to the position of the employees (e.g. lower level to Assistant General Manager to Managing Director). Though private bank gives higher salary and allowances to employees than public banks, but they give lower incentive bonuses and other benefits than the public banks, which de-motivates employees in private sectors. Table 8 explains, both types of banks do not provide any recreational or entertainment allowance but unlike public banks, private banks provide benefit in a form of yearly paid vacation. Although there is no specific fund for short term disability in both banks, public banks support from the general fund, bear the cost if this occurs in workplace and maintain the cost till retirement. Despite these facilities if an employee is not physically fit then bank applies forced retirement. In Private Banks employee can take sick leave or casual leave. If an employee dies on the job, his/her family gets BDT 30000 in public banks. In case of abscond with pay, both companies follow a minimum 1 to 2 months of prior leaving notice as well as custodian services from the employee after quitting.

TABLE 9: h) Industrial Relations Criteria:

\begin{tabular}{|c|c|c|c|c|c|c|c|}
\hline \multicolumn{8}{|c|}{ 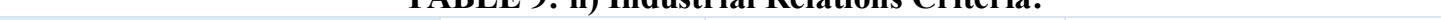 } \\
\hline & \multirow{3}{*}{ Industrial relations criteria } & \multirow{2}{*}{\multicolumn{2}{|c|}{$\begin{array}{c}\text { 1971-1980 } \\
\text { Overall Industries }\end{array}$}} & \multirow{2}{*}{\multicolumn{2}{|c|}{$\begin{array}{c}\text { 1981-1990 } \\
\text { Overall Industries }\end{array}$}} & \multirow{2}{*}{\multicolumn{2}{|c|}{$\begin{array}{c}\text { 1991-2010 } \\
\text { Overall Industries }\end{array}$}} \\
\hline & & & & & & & \\
\hline & & Public & Pvt. & Public & Private & Public & Private \\
\hline & $\begin{array}{l}\text { The labor union is highly trusted } \\
\text { by members of the company }\end{array}$ & $\begin{array}{l}\text { Not } \\
\text { much }\end{array}$ & - & Not satisfied & $\begin{array}{l}\text { No labor or } \\
\text { officer } \\
\text { union }\end{array}$ & $\begin{array}{l}\text { Weak } \\
\text { relationship }\end{array}$ & $\begin{array}{l}\text { No labor or } \\
\text { officer } \\
\text { union }\end{array}$ \\
\hline 2 & $\begin{array}{l}\text { Labor union in this company } \\
\text { more politically active }\end{array}$ & $\begin{array}{l}\text { Highly } \\
\text { active }\end{array}$ & - & $\begin{array}{l}\text { Influenced } \\
\text { much }\end{array}$ & NA & $\begin{array}{l}\text { Significantly } \\
\text { influenced }\end{array}$ & NA \\
\hline 3 & $\begin{array}{c}\text { Labor union in this company is } \\
\text { working hard with management } \\
\text { to solve problem at the work } \\
\text { place }\end{array}$ & $\begin{array}{l}\text { Weakly } \\
\text { organize } \\
\text { d, }\end{array}$ & - & self centered & NA & $\begin{array}{l}\text { Less focused on } \\
\text { employee } \\
\text { welfare }\end{array}$ & NA \\
\hline
\end{tabular}


Industrial Relations Criteria: Public banks allow labor union which refers to as Collective Bargaining Agent (CBA). It is organized by both the representative of the employees and employees. But CBA is weakly organized, and they are more self-interested unions. They have a little influence in rising issues like - the increasing benefits, compensation among the employees. On the other hand Table 9 showed private banks do not have any labor union in their organization. Both the banks have weekly schedule which indicates their upcoming targets, current track, need of improvement, and suggestions. However none of the banks follow those suggestions mostly.

TABLE 10: Company performance

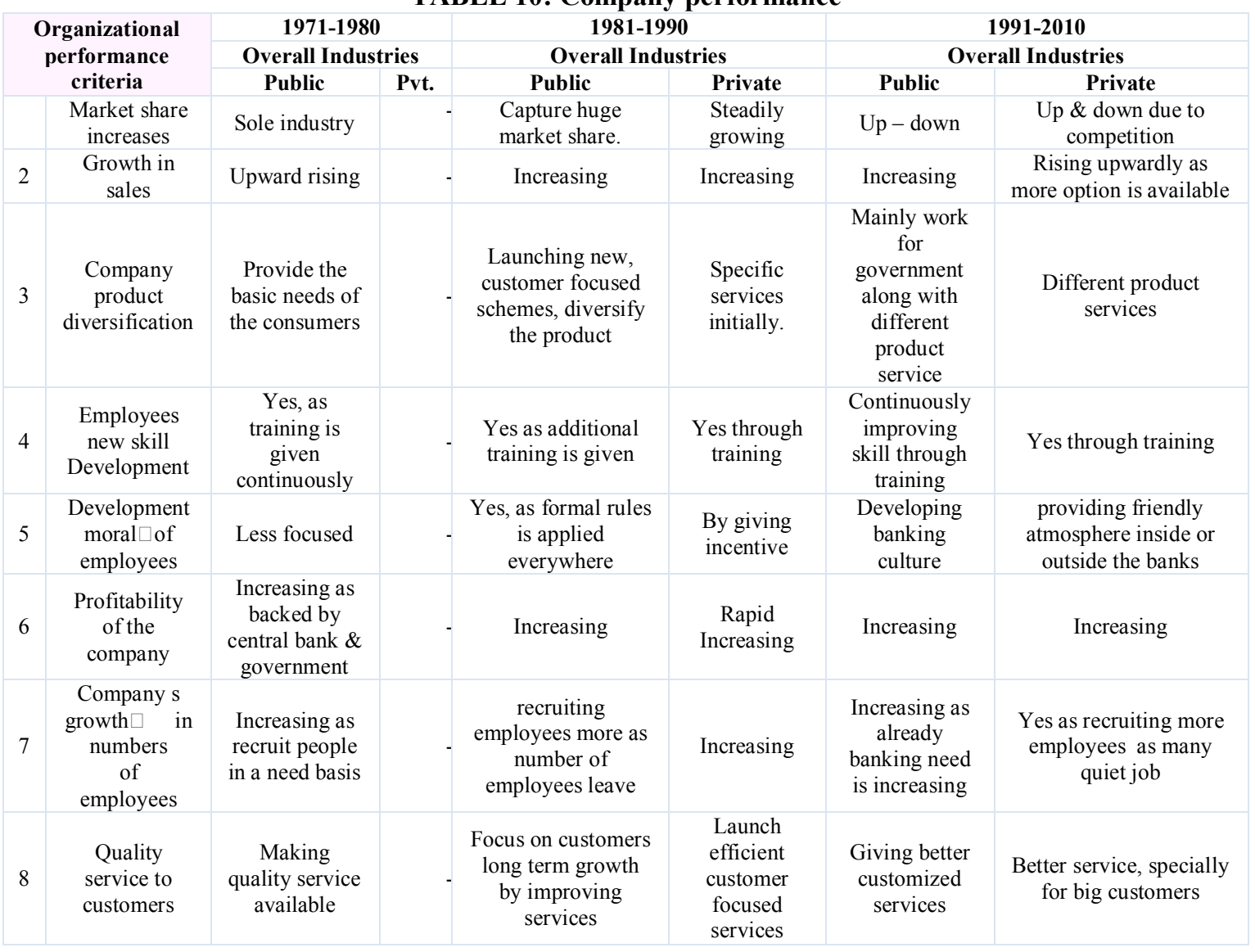

Organizational performance criteria: the overall performance and development of the organization has increased because of increased employee skill and because of the blend of fresh recruiters \& experienced ones as demanded by technological change which is stated in Table 10. Employee ethics developed as companies give incentive or provide friendly atmosphere inside the banks. Corruption is a prevailing incidence in Bangladesh. It is noticeable that though both types of banks are backed by the corrupted and powerful people in terms of fixing - recruitment, salary payment or promotion, but the percentage of unethical acts are lower in public banks. Growth in sales is upwardly rising in both public and private banks as online and other beneficial packages are available by the banks. 


\section{Proposed HR Module of paradigm shift}

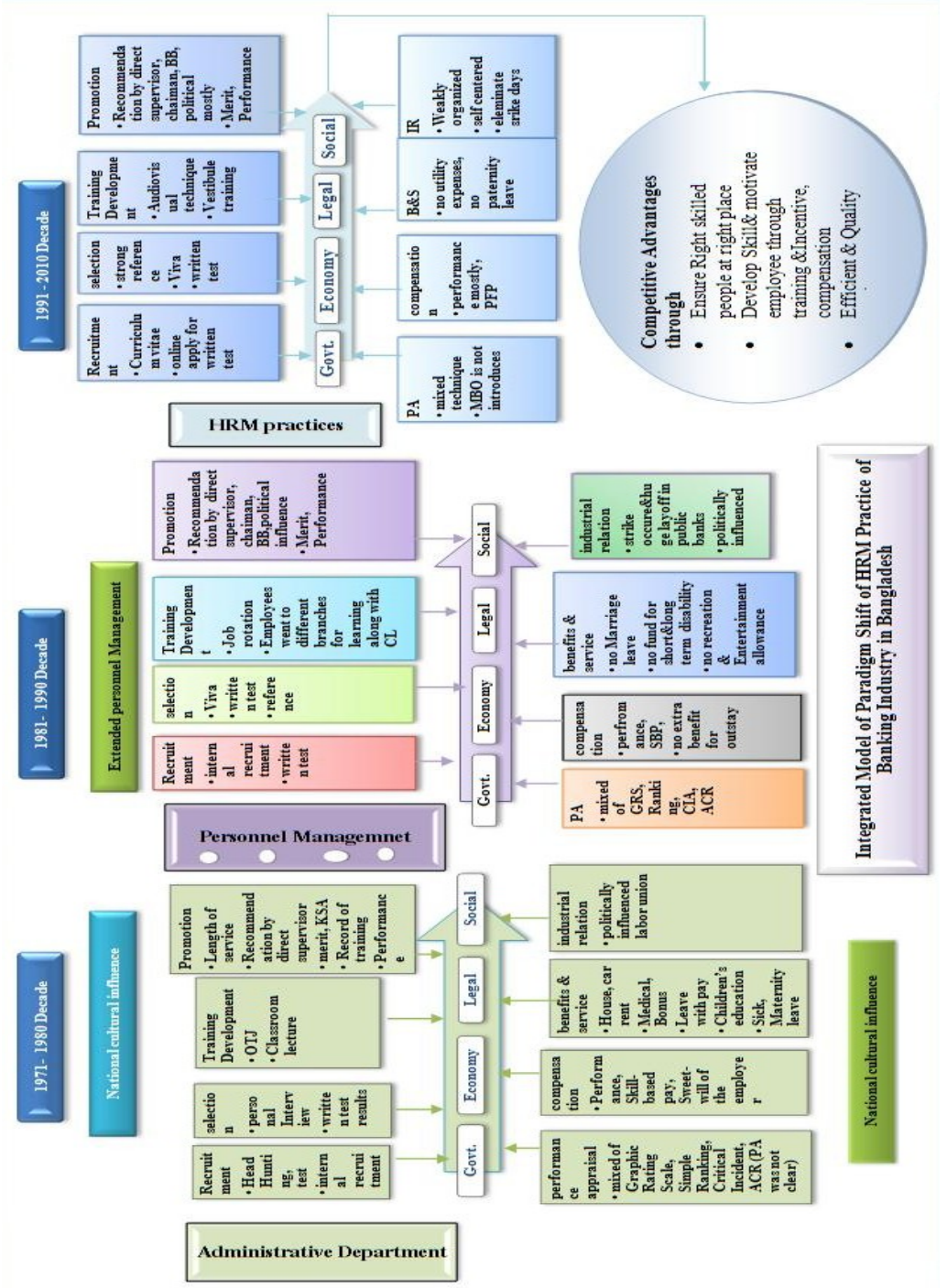

\section{HR Module of paradigm shift}

From our study we developed a module of paradigm shift above to explore the banking industry in Bangladesh, and to explore the administrative department who were mainly involved in hiring, firing and letter issuance-dispatching-filing stage [18]. They were not concerned to give the required training to increase the efficiency or to provide compensation package, benefits or incentive which will motivate the employees to perform better, and thus increase the profitability of the banks. During the period of $1971-1980$, banking industry were challenged significantly in terms of the government, socio economical, legal and national cultural sector which influence the growth of banking industry [25].

Banking industry plays an important role in our free market economy and covers all the spheres of the economy. Our country has made remarkable development and growth in some major areas including the social sector and achieved relative fiscal stability over the decade since its independence except after the September, 2000 terror which led the economic condition to uncertainties, and impacted banking industry extremely [12].

We scrutinize the fact that Bangladesh mainly fit into the hierarchical society according to Hofstede's cultural dimension \& there is a high degree of power distance [5]. Still, there is no direct communication between the top level management and employees. Decision flows top to bottom; suggestion of the employees is listened during the weekly meeting, but did not enforce those and banking industry did not practice MBO (Management by Objective) [26] [27]. Regarding the recruitment procedure, Bangladesh, by its social set-up, encourages strong relationship which influences recruitment. In such a set up, internal recruitment was seen along with head hunting during the year 1971 - 1990, and this practice is still found at the present time. During 1991 - 2010, online application was introduced in both sectors for MTO (Management Trainee Officer) post and it has increased the efficiency in recruitment by saving energy and time instead of recruiting from the stored 
CV for suitable position. In banking industry employees are recruited for the MTO post. Middle and senior levels vacant posts are filled through succession planning process from the existing employee pool. But sometimes deserving candidates are deprived of promotion due to the political influence and chairman's recommendation [28]

After analyzing the study, we gained the information that in 1971 - 1980, Public banks practiced OTJ (On the Job Training) and class lecture for the training and development purpose with the co-operation of the administrative department and the personnel management. The year 1981 - 1990 did not bring any improved techniques in the banking industry. During the era of 1991 - 2010, Human Resource Management sent employees to different branches during the training period to understand and learn the work of the public banks more clearly and practically. This was enforced till 1998, along with the traditional learning. But after 2008, it was stopped as it was time consuming and new employees were directly sent to the specific branches for their job [29].

Due to the huge technological change, in the last decade banking industry provided some additional training tool to teach the employees new things to perform better and increased the use of latest modern equipments [28]. The determination of compensation, incentives, and benefits in the banking sector of Bangladesh is based on the industry standards and the pay structure. Though the compensation offered by the banks should be based on the individual performance and merit etc. but sometimes the banks determine the compensation without having an actual measurement of the performance. During the time of $1971-2010$, they had no fixed technique of performance measurement, instead of that they used mixed performance appraisal techniques. A huge change is required in the benefits and services areas to reduce the turnover and motivate employees in the public sector [29].

In terms of salary payment, there is a huge difference between the public and private bank. Public banks are giving fewer amounts of salary than the private banks as the compensation. In case of public banks, salary and benefits are approved by the finance ministry which pays less attention to the employee needs. This issue is consequently de-motivating the employees of the public banks. During $1971-1990$, banking industry followed Industrial Relations Ordinance 1969 to maintain good relationship with employees through trade union [12]. But personnel management failed to do so as strikes formed by the trade union which was referred to CBA in 1984 and huge number of employees were fired during that time. The banking industry started to eliminate the strike days through the HRM practices from the era of 1991 - 2010 and now they follow Bangladesh Labor Act, 2006, but still there is a weak relationship between CBA and the banks. CBA is now weakly organized, self centered and significantly politically influenced, and less focused on employee welfare [26] [29].

The practice of job switch is still prevailing in the banking sector of Bangladesh due to the pay structure, which is the prime reason behind the dissatisfaction of the workers [8]. But the turnover rate was relatively low during the year 1991 - 2010, though job enrichment facility is still not updated in banking industry.

Still we have seen that the HR department is very much concerned with the discipline that it set up in the banking industry during the year $1991-2010$. It has enforced strict rules and regulations for each and every aspect of banking, even for non-banking purposes; i.e. the Dress Code. All these major personnel functions of the banking sector are integrated in the best possible way to gain higher productivity. HR roles are now focused on employee motivation, development, retention, and facilitation of organizational development initiatives etc.

After analyzing the study, we found that during the era of 1991 - 2010, the HR managers of the banking sector realized the increased need of people development. Because at that time they identified that factors like deficient executive and staff banking skills; lack of professionalism, accountability and incentives; political patronage; troublesome union activity; and poor IT/MIS skills was hindering the efficiency and costeffectiveness of the operation [13]. To reduce inefficiency and to develop the banking culture and skilled employees, HR department emphasized on providing extensive training along with recruiting right people for the right place, developing skill and motivating employees through proper incentives and benefits. But significant improvement is still needed to gain competitive advantages.

\section{Future implications and Contribution of the study}

In Bangladesh 'the concept of HR' is not very old, still practically it is a new concept and significant research is to be done. But within the last decades, it is widely practiced and now maximum large companies build department of Human Resource Management. As we move into the 'new economy', the focus on people and knowledge has steadily increased. Today, companies face new challenges in dealing with issues such as the scarcity of talent, the value of ideas/knowledge and the changing preferences of the labor market. Consequently, successful HR strategies are needed more than ever [13] [30].

Our study reveals that new millennium has brought about new challenges for HR departments and practitioners around the world. These new challenges raise questions about the nature of HR practices and the role of HR professionals along with involving new approaches for the HR function in product and service 
deliver [30]. Organizations recognize the importance of human capital for competitiveness and ultimate survival in the modern era.

From the study we have learned that there was a huge improvement in the banking industry during last decades. At present this industry has become relatively conscious in maintaining an effective and efficient human resource through HRM which must play a more strategic role in the success of an organization. The role of the HR Manager is evolving with the change in the competitive market environment. Bangladesh has huge potential capacity, but employees need continuous evaluation, motivation as well as mentoring by well planned HR management tactics. So developing HR department is necessary for the banking sector.

But the result of the study also authenticates that though the HRM practices in the banking sector of Bangladesh is improving but the progress rate is very slow. Modernization of HRM as par the employee needs will be a lengthy procedure. Due to the outdated HR practices, the productivity and motivation level of the employees of Bangladesh are very low [3].

Our study explored that biasness affects more or less all the HRM practices in the banking sector. Due to practicing unethical act, in both public and private banks, non deserving candidates deprive the deserving ones. But there are several issues found from the study which are not in accord with other researcher's study, like Ernst \& Young stated that governmental organizations in Bangladesh have become nearly dysfunctional due to biasness and in most training sessions and workshops, non deserving employees are given opportunity because of family ties, political influence or just merely being the favorite of the boss [6], but we found that this scenario is mostly visible in the private banking sector. Training in public banks is less influenced by political and top level management.

The study also revealed that HRM in our country is evolving and is fulfilling their responsibilities to achieve the company's ultimate goal by motivating the employees [10]. On the other hand, organizations that do not emphasis on attracting and retaining talents may find themselves in terrible consequences, as their competitors may be outplaying them in the strategic employment. In order to succeed, HR must be a business driven function with a thorough understanding of the organization's big picture and be able to influence key decisions and policies (e.g. recruiting \& selection, rewards, promotion). In case of selecting the final employees, no one can realize who will be the perfect choice for their position except the HR people. So to ensure recruiting the right people at the right time for the right position HR department should be involved in the final recruiting decision making panel.

Consistently our empirical findings show that with the increased competition worldwide, organizations must become more adaptable, flexible, responsive, and customer-focused to succeed. HR professional has to evolve as a strategic partner, as an employee advocate and as a change mentor within the organization. In general, today's HR Manager mainly focuses on the strategic personnel retention and talent development [30]. So it can definitely be said that, the HR professionals can be coaches, counselors, mentors, and succession planners to help motivate organization's members and their loyalty. In addition to that, the HR managers can also promote and fight for the values, ethics, beliefs, and spirituality within their organizations.

What we found from the study regarding the compensation which is a vital topic in motivating the employees in the banking industry of Bangladesh is that, employees are commonly low paid with little or no extra benefit [7]. Consequently this is de-motivating the employees. So in future the banking sector needs to modify the overall pay structure, (appropriate basic salary, compensation, benefits, incentives). Public banks also need to modify the basic salary which is comparatively very low compared to the private banks, whereas private banks should bring in more benefits \& services. Otherwise the turnover rate which is low now will increase due to the lack of proper initiative on time.

Our Study successfully pulls off the unique thing practiced by the public banks that if promotion is not given to the deserving employees, they raise the salary just to motivate them. But this application is not fair to the deserving employees as promotion might boost their performance and motivation more than the salary increase. But also there are a huge number of employees who are ready to sacrifice quick promotion, job security, and friendly colleagues for higher pay and fringe benefits. Thus, the study challenged the western belief of motivating workers more by the essential rewards like self-sufficiency, and task identity, unless salary and benefits met the basic needs, the intrinsic rewards might not work in Bangladesh [8].

Findings of the study reveal that in Bangladesh trade union is not encouraged in private sector. But public banks allow trade union. So for their own benefit, the public banks should make their trade union more effective and employee focused. The main goal of practicing the trade union includes increasing productivity, quality of goods and services, labor costs, quality of the workforce and motivation by preventing disputes. So, private Banks should allow trade union which might be beneficial to motivate employees and increase efficiency. 


\section{Conclusions}

Though there are several structural and cultural differences between the public and private banking industry, till some major problems in some areas like human resource planning and implementation problem, compensation and benefits criteria, conspiracy in giving promotion and other problem similar to most of the countries are identified. Till banking industry is improving along with the change in the world and other sectors in Bangladesh, strengthening human resource practices in a suitable way.

This paper gave us an insight on how the Banking sector work environment operates. What labor law they are following, how they maintain their relationship with potential employees as well as what is their working environment, is it friendly or less focused on employee satisfaction or not. It gave us an insight on how the people of banking sector are recruited, selected and trained before and after getting into a job, depending on certain important factors. Basically how the overall human resource management practices are carried here.

We also found that the banking sector is providing several motivational factors to satisfy the employees. Still the private banks need to be more focused on the basic employee needs and public banks need to raise their salary structure [7]. The banking sector needs to utilize the new talents by providing better training facility and mentoring them well, and thus they can properly utilize their skills. Experienced employees also have values and lots of potentiality in this sector. So it is necessary to combine these old and new employees' potential skills through training, motivation.

Private Banks mostly call for exam from the posted CV to save time, energy, money in the recruiting where public banks lack in this area. So, Public bank should consider collecting and storing of potential candidates curriculum vitae which help to cope up with the changing world. Public banks are more concern about giving proper training facility to increase skill. Vestibule training which is used as additional training tool for employees to learn new things to perform better in the workplace is practiced in the public banks widely then in private banks. This is more effective and motivates employees that their skills and work are also valued and any kind of skill deficiency regarding technological change is considered and provided appropriate training to overcome it and be productive again in the bank [28].

Training tool like job rotation is not followed properly in banking industry. One employee remains in the same position for long time and in private banks it's practiced only for the MTO position mildly. So need to do job rotations on a regular basis to increase skills, experience and diversified rather than trap in one specified job responsibilities.

Public bank and private banks is different in pay structure as public is influenced by the government and private banks follow their individual pay structure. Due to different structure and having limited resources, there is a huge difference in compensation and benefit area. Private Banks need to give more and more financial incentives to motivate the employees.

In case of final increment, promotion, compensation decision, it is decided \& approved by the top level management; HRD is not using their skills fully. Both banking industry need to improve the policy regarding promotion, compensation and benefits, performance appraisal techniques needs clarification and think alternative way to compensate employees regarding sensitive issues like short \& long run disability, marriage, paternity leave and so on.

Active and efficient trade union help to lower frustration, dissatisfaction, de- motivation among the employees regarding the compensation, promotion, and lay off decision etc [12]. So banking industry should exercise healthy industrial relation, private banks should allow the employees to form labor union. Public banks HR department should maintain relationship with the CBA regarding employee demands and reduce political influence, which will be more beneficial.

Banking industry needs to ensure fairness and transparency in overall human resource management practices like - recruiting, compensation, promotion, training and other areas. Banking industry does not follow the background information checking on a regular basis, which should be done to stop recruiting forged and suspicious candidates. This will help them to maintain less conspiracy and avoid people who are prone to corruption [30].

\section{References}

[1] Chowdhury M. F. (2011). Performance Management Practices in Organizations Operating in Bangladesh: A Deeper Examination. World Review of Business Research, 1(2), Pp. 153 - 167.

[2] Tanure, B., \& Duarte, R. G. (2005). Leveraging competitiveness upon national culture traits: The management of people in Brazilian companies International Journal of Human Resource Management, vol. 16, no.12, pp. 2201-2217.

[3] Akhter, N. (2002). Human resource management in Bangladesh: A study of some local private manufacturing industries. (Unpublished doctoral dissertation). University of Dhaka, Bangladesh

[4] Abdullah A.B.M., Boyle S. \& Joham C. (2011). Cultural Factors in Workforce Management: The Case of Multinational Companies Operating in Bangladesh. International Review of Business Research Papers, 7 (2), pp. 196-211.

[5] Jamil, I. (1998). Administrative Culture in Public Administration: Five Essays on Bangladesh. Bergen: Los- Sentret. Rapport 9801

[6] Ernst \& Young, MCCI, (2007). HR Practices Survey Bangladesh 2006-2007. 
[7] Chowdhury, G. (2000). Review of the book Human resources management practices in Bangladesh. Journal of Business Administration, 26(3\&4), pp. 117-119.

[8] Ali, M 1989, Employee motivation in Bangladesh with special reference to nationalized industrial units located in Rajshahi and Khulna zones. In M. Ahmad \& G. Mclean (Eds.), Bangladesh business research reports (pp.88-94). Dhaka: UGC.

[9] Talukder, A.K.M. (2011), Do Human Resource Practices Lever Organizational Performance? Indus Journal of Management \& Social Sciences, 5(2):106- 114

[10] Moyeen, A \& Huq, A 2001. Human resource management practices in business enterprises in Bangladesh. Journal of Business Studies, Vol. 22, No. 2, pp. 263-270.

[11] K. S. Ali and Dr. N. Khatun. (2009). A Handbook on HR Solution, pp: 7-9.

[12] Faruque, A. A. (2009). Current Status and Evolution of Industrial Relations System in Bangladesh. International Publications, Cornell University, IRL School

[13] Sayeed, Y., Bari, M., Rahman, M., Azad, W., Moniruzzaman, M., \& Shilpi, L. M. (2002) 'Swinging Banking: Not Very Promising'. Bangladesh journal.

[14] Investment portfolio of Janata Bank Limited, (2011). Retrieved December 14, 2011, from http://www.janatabank-bd.com/jb10.htm

[15] Janata Bank Ltd.: Overview. Retrieved December 14, 2011, from (http://www.janatabank-bd.com/jb1.htm).

[16] Janata Bank Ltd.: Summary of employees. Retrieved December 14, 2011, from http://bankinfobd.com/banks/26/Janata Bank

[17] Mutual trust bank ltd: profile, (2011, June 12). Retrieved December 14, 2011, from (http://www.mutualtrustbank.com/about profile.php)

[18] Rahman, S. M. M. (2006). Sonali Bank. Retrieved December 14, 2011, from

[19] Sonali Bank Ltd. Corporate Profile, (2011, November $\quad$ 02). Retrieved $\quad$ December $14, \quad 2011, \quad$ from $\begin{array}{llllll}\text { http://www.sonalibank.com.bd/profile.php } & \\ \text { Sonali Bank Ltd. Overview of the Bank. (2008). Retrieved December 14, from }\end{array}$

[20] Sonali Bank Ltd. Overview of the Bank. (2008). Retrieved December
2011,http://web.archive.org/web/20070703133224/http://www.sonalibank.com.bd/overview of the bank.htm

[21] Perry, C. (1998). Processes of a case study methodology for postgraduate research in marketing. European Journal of Marketing, 32(9/10), 785-802.

[22] Zikmund, W.G. (2000). Business research methods (6th ed.). New York: Dryden Press.

[23] Patton, M.Q. (1990). Qualitative evaluation methods, Beverly Hills, CA: Sage.

[24] Stake, R., (1995). The art of case study research. Thousand Oaks, CA: Sage Publications.

[25] Banking Sector Performance, Regulation and Bank Supervision, (2006). Retrieved December 14, 2011, from http://www.ilocarib.org.tt/Promalco tool/productivity-tools/manual11/m11 $10 . \mathrm{htm}$

[26] Personal Interview with Executive Vice President, Mutual Trust Bank Ltd, $29^{\text {th }}$ March, 2014.

[27] Personal Interview with Management Trainee officer, National Bank Ltd, $16^{\text {th }}$ April, 2014.

[28] Personal Interview with ex - principal officer, Janata Bank Ltd, $25^{\text {th }}$ April, 2014.

[29] Personal Interview with principal officer, Sonali Bank Ltd, $14^{\text {th }}$ March, 2014.

[30] Bhuiyan, A. M. S. (2007, August). Human Resources profession is very challenging in Bangladesh. International Islamic University Chittagong (IIUC), Bangladesh. Retrieved November 20, 2011, from www.greenbangladesh.com. 